\title{
Antituberculosis Drug Reclassification for Proper Management of Rifampicin-resistant and Multidrug-resistant Tuberculosis
}

\section{Suhail Ahmad}

Department of Microbiology, Faculty of Medicine, Health Sciences Center, Kuwait University, Kuwait

*Corresponding author: Suhail Ahmad, Department of Microbiology, Faculty of Medicine, Health Sciences Center, Kuwait University, Kuwait, Tel: 00965-24636503; Email: suhail_ah@hsc.edu.kw

Received date: March 26, 2018; Accepted date: March 31, 2018; Published date: April 06, 2018

Copyright: $@ 2018$ Ahmad S. This is an open-access article distributed under the terms of the Creative Commons Attribution License, which permits unrestricted use, distribution, and reproduction in any medium, provided the original author and source are credited.

\section{Editorial}

Tuberculosis (TB) is a major infectious disease and increasing incidence of drug-resistant-TB is a serious threat to global TB control. Active TB disease in humans is caused mainly by Mycobacterium tuberculosis. Some disease cases are also caused by Mycobacterium africanum (mainly in Africa) and Mycobacterium bovis (due to consumption of unpasteurized milk), two other species belonging to the $M$. tuberculosis complex [1]. The infection is mainly acquired by inhalation of tubercle bacilli expectorated by pulmonary TB patients (open TB) during close human contact [1]. Primary infection either leads to clinically active TB disease or the host immune response arrests multiplication of $M$. tuberculosis. However, complete sterilization is achieved in some individuals only while few bacilli in other individuals escape killing, become dormant and persist in granulomatous lesions (latent TB infection) [1]. The latent infection may remain dormant for a long time or resuscitates to cause active $\mathrm{TB}$, years to decades later, often due to weakening of the host immune response [1]. Nearly $25 \%$ of the world population is latently infected with tubercle bacilli and 5\%-10\% of the infected individuals will eventually develop active $\mathrm{TB}$ disease during their life-time [2]. Reactivation of latent infection is more frequent in people with immunodeficiencies, diabetes, other immunosuppressive conditions or co-infection with human immunodeficiency virus (HIV) [1]. Active TB disease in low TB incidence/high income countries mostly occurs in foreign-born individuals due to reactivation of latent infection while recent infection/re-infection is common in $\mathrm{TB}$ endemic countries $[1,3]$.

Although the incidence of active TB disease and TB deaths are declining, the global burden of TB still remains high. According to the World Health Organization (WHO) annual surveys, there were 10.4 million active TB disease cases in 2016 [4]. Nearly $70 \%$ of disease cased occurred in countries of South-East Asia and Africa and 56\% of cases occurred in China, India, Indonesia, the Philippines and Pakistan [4]. The incidence varied from $<10$ per 100000 populations in highincome countries to $150-300$ per 100000 in most of the 30 high TB burden countries [4]. An estimated 1.7 million people (including 0.37 million HIV-coinfected individuals) died from TB in 2016, making TB as the ninth leading cause of death worldwide and the leading cause of death from a single infectious agent [4]. Most of the TB deaths are now attributed to the resistance of $M$. tuberculosis strains to anti-TB drugs $[4,5]$.

The increasing incidence of DR-TB, multidrug-resistant (MDR)-TB (M. tuberculosis resistant at least to rifampicin and isoniazid, the two most effective first-line drugs) and extensively drug-resistant (XDR)TB (MDR-TB strains additionally resistant to a fluoroquinolone plus injectable agent, kanamycin, amikacin or capreomycin) pose a major threat to global TB control efforts [4]. In 2016, an estimated 600000 new TB cases were resistant to rifampicin (RR-TB) of which 490000 were additionally resistant to isoniazid (MDR-TB) [4]. Nearly $4 \%$ of all new TB cases and 19\% of previously treated cases had MDR-TB and $47 \%$ cases occurred in India, China and the Russian Federation [4]. Patients infected with RR-TB are also treated as MDR-TB cases [4]. Successful treatment of RR-TB and uncomplicated MDR-TB ( $M$. tuberculosis strains resistant only to rifampicin and isoniazid) is higher compared to treatment of MDR-TB resistant to additional first-line drugs [4-6]. Nearly $10 \%$ of all MDR-TB cases are now estimated to have XDR-TB [4,5]. Some (India, Iran, Italy and South Africa) countries have also reported totally drug-resistant (TDR)-TB caused by $M$. tuberculosis strains resistant to all tested anti-TB drugs [5-7]. However, WHO currently does not endorse the definition of TDR-TB since drug susceptibility testing (DST) results for many second-line and other drugs are poorly reproducible [4].

Treatment of fully drug-susceptible-TB is highly efficacious and if implemented properly, has a cure rate of $>90 \%[4,6,8]$. On the contrary, treatment of patients with DR-TB, particularly MDR-TB is much more difficult due to lengthy (12-24 months), more expensive and more toxic drug regimens and the patients often experience clinical failure or disease relapse [5-7]. Recent WHO data show that the treatment success rates for drug-susceptible TB, MDR-TB and XDR-TB are $83 \%$, $54 \%$, and $30 \%$, respectively [4]. Despite the long duration of treatment, the cure rates for MDR-TB are dismally low and untreated MDR-TB leads to XDR-TB [6]. Thirty-five countries, mostly in Asia and Africa have recently introduced short-course (9-12 months) Bangladesh regimen for the treatment of RR-TB/MDR-TB patients, with treatment success rates approaching 90\% [4,9]. Two new anti-TB drugs (bedaquiline and delamanid) have recently been approved to treat MDR-TB [4,10]. More than 80 countries have started using bedaquiline and $>50$ countries have started including delamanid in treatment regimens for MDR-TB [4]. In an effort to improve treatment outcome specifically for RR-TB and MDR-TB, WHO has recently reclassified anti-TB drugs.

The anti-TB drugs were previously divided into 5 groups (Group 1 to 5) based on decreasing efficacy and increasing toxicity. Group 1 (first-line) agents included relatively less toxic and mostly bactericidal (rifampicin, isoniazid, ethambutol and pyrazinamide) oral drugs suitable for combination therapy [8]. Streptomycin is not used routinely anymore due to higher frequency of resistance and the availability of other active drugs suitable for oral therapy [6,8]. Group 2 included injectable aminoglycosides (kanamycin and amikacin) and cyclic polypeptides (capreomycin or viomycin) $[6,8]$. Group 3 included fluoroquinolones, particularly bactericidal agents such as levofloxacin (at high dose), gatifloxacin and moxifloxacin [6,8]. Group 4 included mainly bacteriostatic, less efficacious and more toxic oral agents that 
were used in therapy regimens only for treatment of MDR-TB and XDR-TB $[6,8]$. High dose isoniazid and rifabutin were also used occasionally as second-line oral agents [6,8]. Group 5 included thirdline reinforcing agents that were used only for the treatment of MDR$\mathrm{TB}$ or XDR-TB but were not recommended for routine use due unproven efficacy and serious side effects [6,11]. However, these classifications are now considered inadequate for the proper management of RR-TB and MDR-TB patients since treatment of MDR-TB by conventional approaches for 18-24 months actually amplifies resistance further as successful outcome is achieved in $\sim 50 \%$ of treated patients only [4]. Furthermore, the availability of two new anti-TB drugs, bedaquiline and delamanid, to treat MDR-TB has renewed hope for improved outcome of MDR-TB and to prevent development of XDR-TB [10,12]. Consequently, the WHO has recently reclassified anti-TB drugs with the aim of developing a more efficacious and better tolerated regimen for RR-TB and MDR-TB cases [13]. The currently available drugs, including new drugs (bedaquiline and delamanid), are classified into four groups (Group A to Group D) specifically for the treatment of RR-TB and MDR-TB [13]. The remaining first-line drugs (pyrazinamide and ethambutol) are now assigned to a subclass of Group D agents [13].

Group A includes fluoroquinoloes (moxifloxacin, gatifloxacin or high-dose levofloxacin) as the best agents for the treatment of MDRTB as they have excellent safety profile and bactericidal and sterilizing activity against $M$. tuberculosis. They are now placed ahead of injectable agents as their use is associated with a more favourable outcome [13]. Second-line injectable (amikacin, kanamycin and capreomycin) drugs have now been placed in Group B as they are bactericidal but lack sterilizing activity [13]. According to WHO, the Group B may include in future, three oral drugs; linezolid (or sutezolid or tedizolid), bedaquiline and delamanid, if they prove to be more effective and less toxic than the injectables for an all oral therapy for MDR-TB [13]. Group C currently includes second-line oral drugs; linezolid, clofazimine, ethionamide, prothionamide, cycloserine and terizidone [13]. Linezolid is bactericidal with sterilizing action and the toxicity can be managed by reducing the dose [6]. Ethionamide and prothionamide have moderate bactericidal activity but also exhibit higher toxicity [13]. Clofazimine has some strerilizing activity and good tolerability, however, skin decoloration (drug side effect) may limit its use in some ethnic groups $[4,9,13]$.

Group D drugs have been further dividied into three sub-groups; D1, D2 and D3. Group D1 includes pyrazinamide and two other firstline drugs (ethambutol and high-dose isoniazid) provided they are likely to be effective [13]. Rifabutin may also be considered for some M. tuberculosis isolates with specific rpoB mutations which confer resistance to rifampicin but are susceptible to rifabutin [6,13]. Group D2 includes two new drugs; bedaquiline and delamanid that have recently been approved to treat MDR/XDR-TB cases when no other options are available or other drugs are not tolerated by the patient to complete at least four active drug-regimen [10,12]. Bedaquiline and delamanid are bactericidal with sterilizing activity as they target actively replicating and dormant bacilli $[12,14]$. Bedaquiline has been safely used for up to 18 months and has also been used in combination with delamanid [12,14]. Group D3 includes p-aminosalicylic acid, thiacetazone, amoxycillin-clavulanate, imipenem-clavulanate and meropenem-clavulanate [13]. Meropenem/clavulanate was recently found to be more active than imipenem/clavulanate and is bactericidal [15]. Thus meropenem/clavulanate may also be used as a core drug for pre-XDR/XDR-TB cases with resistance to second-line injectables.
According to the new drug classification proposed by WHO, patients with RR-TB and MDR-TB should be treated with at least five effective TB medicines during the intensive phase and should include pyrazinamide and four core second-line drugs including one drug from Group A, one drug from Group B and at least two drugs from Group C [13]. The remaining first-line drugs (ethambutol, high-dose isoniazid and/or rifabutin) may also be used if they are likely to be beneficial based on drug resistance profile [13]. High-dose isoniazid may be added when a mutation in the $k a t G$ gene is absent, however, it should not be counted as one of four active drugs [13]. Similarly, rifabutin may be added if susceptibility is suggested by a favorable $r p o B$ mutation profile [13], but it should not be counted as one of four active drugs. If sufficient number of effective drugs cannot be composed as given above, an agent from Group D2 and other agents from Group D3 may be added to bring the total to five drugs. If pyrazinamide is compromised due to resistance or can not be used, the regimen may be reinforced with a drug from Group C or Group D2, and if it is not possible then from Group D3 [13]. Other important factors for successful management of RR-TB and management of MDR-TB include easy availability of the drugs at an affordable price, reliable DST facilities and recognizing and promptly managing adverse drug reactions $[4,13]$. The treatment for RR-TB and MDR-TB should last 18 months or longer and should include minimum number of second-line drugs considered to be effective based on patient history or drug-resistance patterns [13].

Another important development is the introduction of a cheaper and shorter 'Bangladesh regimen' of 9-12 months' duration for patients with RR-TB and MDR-TB who were not previously treated with second-line drugs and in whom resistance to fluoroquinolones and second-line injectable agents is either excluded or is considered highly unlikely $[9,3,16]$. This regimen includes an intensive phase of 4 months with high-dose gatifloxacin, pyrazinamide, ethambutol, clofazimine, kanamycin, prothionamide and isoniazid followed by 5 months of continuation phase with high-dose gatifloxacin, pyrazinamide, ethambutol, clofazimine and reported treatment success rate of nearly $90 \%[13,16]$. Gatifloxacin which likely played a critical role in its success was unfortunately withdrawn from the market due to association of this drug with dysglycaemia, depriving resource-poor countries of an efficacious, effective and inexpensive drug [17]. The WHO has now replaced gatifloxacin with moxifloxacin in its guidelines [13]. The revised shorter regimen now includes an initial phase of 4 to 6 months of treatment with pyrazinamide, kanamycin, moxifloxacin, prothionamide, clofazimine, high-dose isoniazid and ethambutol followed by 5 months of continuation phase with pyrazinamide, kanamycin, moxifloxacin and ethambutol $[4,9,13]$.

The WHO guidelines also emphasize the importance of rapid diagnosis of drug resistance detection by molecular testing to ensure appropriate selection of patients who can truly benefit from the shorter MDR-TB regimen [13]. The liquid medium-based fully automated culture systems (e.g. MGIT 960 system) report results within 10-14 days, however, their performance for $M$. tuberculosis isolates carrying specific resistance conferring mutations in target genes for some firstline drugs (e.g. rifampicin and ethambutol) and many second-line drugs is sub-optimal $[18,19]$. Molecular methods are rapid reporting DST results within 1-2 days $[19,20]$. However, the sensitivity of some methods is affected by the frequency of specific mutations in resistance conferring genes which varies in different geographical settings/ethnic groups [21-23]. Whole-genome sequencing is a newer alternative that provides rapid drug resistance profiles for all anti-TB drugs to inform treatment within 1-2 days [24,25]. Rapid diagnosis also reduces the 
duration of infectious period by rapid initiation of treatment with an adequate regimen, further transmission of MDR-TB within the community and development of additional resistance leading to preXDR-TB and XDR-TB $[4,6]$.

\section{Acknowledgements}

Research support provided by Kuwait University Research Sector through grant MI03/16 is gratefully acknowledged.

\section{References}

1. Ahmad S (2010) New approaches in the diagnosis and treatment of latent tuberculosis infection. Respir Res 11: 169.

2. Houben RM, Dodd PJ (2016) The global burden of latent tuberculosis infection: a re-estimation using mathematical modelling. PLoS Med 13: e1002152.

3. Mokaddas E, Ahmad, S, Samir I (2008) Secular trends in susceptibility patterns of Mycobacterium tuberculosis isolates in Kuwait, 1996-2005. Int J Tuberc Lung Dis 12: 319-325.

4. World Health Organization (2017) Global tuberculosis report 2017 Geneva, Switzerland.

5. Dheda K, Gumbo T, Gandhi NR, Murray M, Theron G, et al. (2014) Global control of tuberculosis: from extensively drug-resistant to untreatable tuberculosis. Lancet Respir Med 2: 321-338.

6. Ahmad S, Mokaddas E (2014) Current status and future trends in the diagnosis and treatment of drug-susceptible and multidrug-resistant tuberculosis. J Infect Public Health 7: 75-91.

7. Dheda K, Gumbo T, Maartens G, Dooley KE, McNerney R, et al. (2017) The epidemiology, pathogenesis, transmission, diagnosis, and management of multidrug-resistant, extensively drug-resistant, and incurable tuberculosis. Lancet Respir Med 5: 291-360.

8. Mitchison D, Davies G (2012) The chemotherapy of tuberculosis: past, present and future. Int J Tuberc Lung Dis 16: 724-732.

9. Sotgiu G, Migliori GB (2017) Effect of the short-course regimen on the global epidemic of multidrug-resistant tuberculosis. Lancet Respir Med 5 159-161.

10. Zumla A, Memish ZA, Maeurer M, Bates M, Mwaba P, et al. (2014) Emerging novel and antimicrobial-resistant respiratory tract infections new drug development and therapeutic options. Lancet Infect Dis 14: 1136-1149.

11. Dooley KE, Obuku EA, Durakovic N, Belitsky V, Mitnick C, et al. (2013) World Health Organization group 5 drugs for the treatment of drugresistant tuberculosis: unclear efficacy or untapped potential? J Infect Dis 207: 1352-1358

12. Lewis JM, Hine P, Walker J, Khoo SH, Taegtmeyer M, et al. (2016) First experience of effectiveness and safety of bedaquiline for 18 months within an optimized regimen for XDR-TB. Eur Respir J 47: 1581-1584.
13. World Health Organization (2016) WHO treatment guidelines for drugresistant tuberculosis 2016 update. Geneva, Switzerland

14. Tadolini M, Lingtsang RD, Tiberi S, Enwerem M, D’Ambrosio L, et al. (2016) First case of extensively drug-resistant tuberculosis treated with both delamanid and bedaquiline. Eur Resp J 48: 935-938.

15. Tiberi S, Sotgiu G, D’Ambrosio L, Centis R, Abdo Arbex M, et al. (2016) Comparison of effectiveness and safety of imipenem/clavalunate-versus meropenem/clavalunate-containing regimens in the treatment of multidrug and extensively drug-resistant tuberculosis. Eur Respir J 47: 1758-1766.

16. Van Deun A, Maug AK, Salim MA, Das PK, Sarker MR, et al. (2010) Short, highly effective and inexpensive standardised treatment of multidrug-resistant tuberculosis. Am J Resp Crit Care Med 182: 684-692.

17. Chiang CY, Van Deun A, Rieder HL (2016) Gatifloxacin for short, effective treatment of multidrug-resistant tuberculosis. Int J Tuberc Lung Dis 20: 1143-1147.

18. Ahmad S, Mokaddas E, Al-Mutairi N, Eldeen HS, Mohammadi S (2016) Discordance across phenotypic and molecular methods for drug susceptibility testing of drug-resistant Mycobacterium tuberculosis isolates in a low TB incidence country. PLoS One 11: e0153563.

19. Schön T, Miotto P, Köser CU, Viveiros M, Böttger E, et al. (2017) Mycobacterium tuberculosis drug-resistance testing: challenges, recent developments and perspectives. Clin Microbiol Infect 23: 154-160.

20. Al-Mutairi N, Ahmad S, Mokaddas E (2011) Performance comparison of four methods for rapid detection of multidrug-resistant Mycobacterium tuberculosis strains. Int J Tuberc Lung Dis 15: 110-115.

21. Abal AT, Ahmad S, Mokaddas E (2002) Variations in the occurrence of the S315T mutation within the katG gene in isoniazid-resistant clinical Mycobacterium tuberculosis isolates from Kuwait. Microb Drug Resist 8: 99-105.

22. Ahmad S, Fares E, Araj GF, Chugh TD, Mustafa AS (2002) Prevalence of S315T mutation within the katG gene in isoniazid-resistant clinical Mycobacterium tuberculosis isolates from Dubai and Beirut. Int J Tuberc Lung Dis 6: 920-926.

23. Ahmad S, Al-Mutairi NM, Mokaddas E (2012) Variations in the occurrence of specific rpoB mutations in rifampicin-resistant Mycobacterium tuberculosis isolates from patients of different ethnic groups in Kuwait. Indian J Med Res 135: 756-762.

24. Brown AC, Bryant JM, Einer-Jensen K, Holdstock J, Houniet DT, et al. (2015) Rapid whole-genome sequencing of Mycobacterium tuberculosis isolates directly from clinical samples. J Clin Microbiol 53: 2230-2237.

25. Votintseva AA, Bradley P, Pankhurst L, Del Ojo Elias C, Loose M, et al. (2017) Same-day diagnostic and surveillance data for tuberculosis via whole-genome sequencing of direct respiratory samples. J Clin Microbiol 55: $1285-1298$. 